\title{
THE ANTI-INFLAMMATORY EFFECT OF GARDI PATCH IN CARRAGEENAN-INDUCED PAW EDEMA OF RATS
}

\author{
Dejidmaa B. *, Uuganbayar B., Erdenechimeg Ch., Chimedragchaa Ch., Munkhzul G. \\ Institute of Traditional medicine and Technology, Ulaanbaatar, Mongolia \\ *corresponding author, e-mail: b.dejidmaa@yahoo.com
}

ARTICLE INFO: Received: 10 Nov, 2017; Revised: 22 Dec, 2017; Accepted: 25 Dec, 2017

\begin{abstract}
Gardi -5 has been used in traditional Mongolian medicine as an analgesic and antibacterial medicine. The drug has been studied in chemical-pharmacological research. We have a new natural patch from the prepared Gardi-5. The present work was undertaken to evaluate the traditional drug Gardi patch for its anti-inflammatory activity.

The method of Miho Sekiquichi was used to study acute inflammation. Rats in groups of five each were treated with vehicle, Gardi patch and Ketoprofen patch one hour prior to Carrageenan injection. $0.1 \mathrm{ml}$ of $1 \%$ Carrageenan was injected into the sub plantar tissue of left hind paw of each rat. Swelling of carrageenan injected foot was measured at 0, 30, 60, 120, 180, 240, 300 mins using Plethysmometer (UGO Basile, Italy). The right hind paw was injected with $0.1 \mathrm{ml}$ of vehicle.

The Gardi patch significantly $(p=0.000)$ inhibited carrageenan induced rat paw edema as compared to control group. In assay data, the TNF- $\alpha, P G E 2$ secretion in serum were highly elevated by carrageenan induction but administration of Gardi patch significantly reduced serum secretion of inflammatory mediators as compared to vehicle group. Gardi patch has an anti-inflammatory properties.
\end{abstract}

Keywords: Gardi patch; traditional Mongolian medicine; carrageenan; anti-inflammatory effect; Wistar rat;

\section{INTRODUCTION}

Garidi-5 has been used in traditional Mongolian medicine as an antibacterial and analgesic agent for treatments of various diseases including typhus, dyphteria, joint conditions, neurological and skin disorders. Garidi-5 is composed of 5 herbs including Terminalia chebula Retz., Aconitum Kusnezoffii Reichb., Acorus calamus L.,Saussurea lappa L., and musk of Moschus moschiferus.[1,2] As written in traditional medical sourcebooks the compounds of Gairidi-5 mainly balance humors, detoxify the body, kill bacteria and relieve pain [3]. The drug has been studied chemical-pharmacological research. We have a new natural patch from the prepared Gardi-5.

In this study, using the carrageenaninduced rat paw pad edema model, we evaluated the anti-inflammatory effect of a $10 \%$ Gardi patch. The effect of the Gardi patch was compared to with commercially available $3.75 \%$ Ketoprofen-containing patches. 


\section{MATERIAL AND METHODS}

Animals: A total male 50 Wistar rats (180$220 \mathrm{~g})$ were used for the study and all were housed in a quiet room with $12 \mathrm{~h}$ light/dark cycle. All experiments were approved by the Animal Studies Committee at Institute of Traditional medicine and technology of Mongolia.

Material: Gardi patch produced in of Institute of Traditional Medicine and Technology of Mongolia, was used in this study.

Anti-Inflammatory Study. Wistar male rats (aged 5 weeks) were measured for the volume of the right hind footpad paw in advance with the paw volume-measuring instrument, and 50 randomly selected rats were assigned to groups (10 animals in each group). The measured paw volumes were handled as the baseline values. In each study, a piece of drug patch was applied at the back center of the right hind footpad paw of each rat and fixed with Band-Aid.

During the treatment, the drug patch was removed at 2 hours after application, and $0.1 \mathrm{~mL}$ of an aqueous $1 \mathrm{w} / \mathrm{v} \%$ suspension of $\lambda$-carrageenan in physiological saline was injected subcutaneously into the right hind footpad paw pad as an inflammationinducing agent. In the inflammation control group, no drug patch was applied, and only the inflammation-inducing agent was administered. At $0,30,60,120,180,240,300$ minutes after the induction of inflammation, the paw volume was measured, and the edema rate (\%) was calculated using the baseline value. Swelling of carrageenan injected foot was measured at $0,30,60,120,180,240$,
300 min using Plethysmometer (UGO Basile, Italy). Furthermore, efficacy of treatment with use of $1 \%$ carrageenan was found according to PGE2 and TNF $\alpha$ level. Blood samples were collected via cardiac puncture at $0,30,60$, $120,180,240,300$ after the administration of carrageenan and from healthy rats.

All rats were euthanized with phenobarbital sodium before blood collection. The collected blood samples were centrifuged at $377.3 \mathrm{~g}$ for 10 min at $4^{\circ} \mathrm{C}$, and the plasma supernatant was stored at $-20^{\circ} \mathrm{C}$ until further analysis. The plasma levels of TNF-a and PGE2 were detected using solid-phase sandwich enzymelinked immune sorbent assay (ELISA, Shanghai MLBIO Biotechnology Co. Ltd.) kits specific for the detection of these factors, and the absorbance was measured at $450 \mathrm{~nm}$ by a plate reader (Chromate 4300 micro plate, Shanghai MLBIO Biotechnology Co. Ltd., China).

In the study used $10 \%(100 \mathrm{mg})$ Gardi patch, 3.5\% (133 mg) Ketoprofen patch (Patex ${ }^{\circledR}$, Nipro Patch Co., Ltd). Since the density of each drug imbedded in the sheet differed, the applied dose was controlled by standardizing sheet size in this study at $2.0 \mathrm{~cm} \times 1.75 \mathrm{~cm}$, based on the relative difference between human and rat body sizes [4-6].

\section{Statistical analysis}

Data are reported as means \pm SD. Statistical significance was determined by one-way analysis of variance followed by Tukey's multiple comparison test. A P value 0.05 was considered statistically significant.

\section{RESULTS}

Carrageenan induced rat paw edema

In the control group, the edema rate increased with time after induction of

inflammation with a maximum at 5-6 hours (Figure 1). 


\section{Footpad edema}

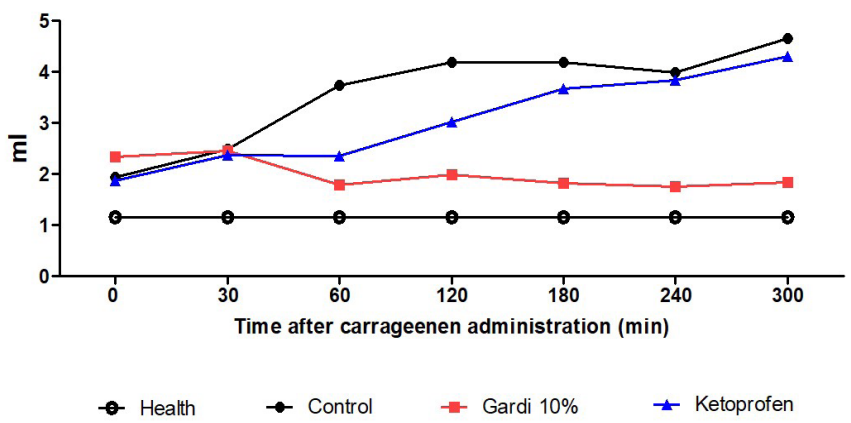

Figure 1. Anti-inflammatory effect (AUEC: Area under the effect curve) of 10\% diclofenac patch or other NSAID patch on the carrageenan-induced hind footpad paw edema in rats

In the Gardi patch group, the edema rates were lower than those in the inflammation control group as well as in the Ketoprofen group from 1 hour after induction of inflammation, and a significant difference was seen between the Gardi patch group and the inflammation control group $(\mathrm{p}=0.009)$ at 6 hours after induction of inflammation.
Effect of Gardi patch on the expression of pro-inflammatory cytokines of plasma,

Effect of Gardi patch on the expression of pro-inflammatory cytokines of plasma. In the control group, the levels PGE2 significantly increased after carrageenan administration and reached peak levels at $300 \mathrm{~min}$.
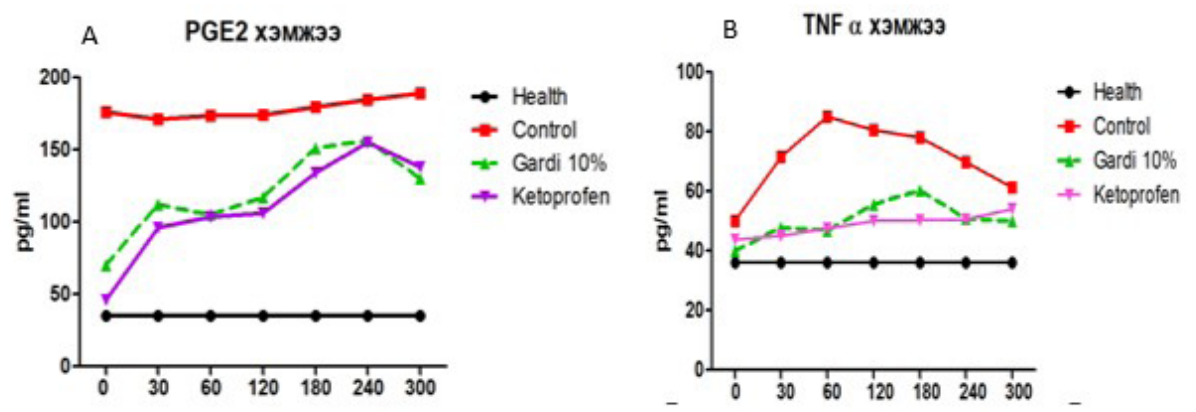

Figure 2. Changes in the levels of pro-inflammatory cytokines

A, Interleukin (IL-6); B, tumor necrosis factor (TNF a)

However, the levels of the late stage proinflammatory cytokine TNF-a increased gradually and reached a peak at $60 \mathrm{~min}$. In contrast, the rats treated with Gardi patch had significantly lower levels of PGE , (Carrageenan+Gardi patch group vs
Carrageenan group: $\mathrm{p}=0.05$ at 60,120 and 240 $\min )$ and of TNF-a ( $p=0.05$ at 120, 180, 240, and $300 \mathrm{~min}$ ) (Figure 2). Statistical analysis revealed that Gardi patch and Ketoprofen significantly inhibited the development of edema 0-300 min after treatment (Fig. 2) and they both showed anti-inflammatory effects in Carrageenan induced rat paw edema. 


\section{DISCUSSION}

This study was performed to evaluate the putative anti-inflammatory activities of Gardi patch, using various animal model to inflammation effects. The injection of Carrageenan into mice produces a typical biphasic edema associated with the production of several inflammatory mediators, including bradykinin, prostaglandins and cytokines. The first phase peaks at $3 \mathrm{~h}$ and the delayed phase peaks at $48 \mathrm{~h}$ after Carrageenan injection [7.8]. Development of edema induced by Carrageenan is commonly correlated with the early exudates stage of inflammation, which is one of the important processes of inflammatory pathology [9.10].

It was found that the injection of Carrageenan into the mice paw induced the liberation of bradykinin, which later induced

\section{CONCLUSION}

The results of this study showed that, in addition to the potent PGE2 and TNF $\alpha$ inhibiting activity and the Gardi patch the biosynthesis of PGE2 and other autacoids that are responsible for the formation of the inflammatory exudates [11-14]. We also evaluated the anti-inflammatory effects of Gardi patch on paw edema induced by Carrageenan in mice and detected the levels of TNF- $\alpha$ and PGE2 the paw edema 0-300 min after Carrageenan injection. It is clear from (figure 2).

Statistical analysis revealed that Gardi patch and Ketoprofen significantly inhibited the development of edema 0-300 min after treatment (Fig. 2) and they both showed antiinflammatory effects in Carrageenan induced rat paw edema. In the current study, the levels of TNF- $\alpha$ and PGE2 were decreased significantly of Gardi patch.

was effective in decrease paw edema in Carrageenan induced rat paw edema model. Gardi patch has an anti-inflammatory effects. 


\section{REFERENCES}

1. Hang $G$ Z. The studies on Guality standart of Mongolian Medicine Garidi-5 Pills, Department of traditional Mongolian medicine, Inner Mongolia Medical College, Hohhot China. 2011.

2. Dagvatseren B., Khishigjargal S., Narantsetseg G., at all. A reference book of traditional drug and medicinal materials. Ulaanbaatar, Mongolia. Traditional Medical Science, Technology and Production Corporation.2003;209-210.

3. Tumurbaatar N, Khatanbaatar Z, Tserendagva D. An introduction to Mongolian traditional medicine. Ulaanbaatar: Munkhiin Useg; 2006.

4. Miho Sekiguchi, Masayoshi Shirasaka. Analgesic effect of percutaneously absorbed nonsteroidal anti-inflammatory drugs: an experimental study in a rat acute inflammation model. BMC Musculoskeletal Disorders 2008, 9:15

5. Kozo Takayama, Akihiko Hirose. Comparison of the Anti-Inflammatory and Analgesic Effects in Rats of Diclofenac-Sodium, Felbinac and Indomethacin Patches. Int J Biomed Sci 2011; 7 (3): 222-229

6. Uuganbayar B, Sugarjav E, Ariunaa Z, Oyun Z, Molor-Erdene P, Chimedragchaa Ch, Oldokh $S$, Davaakhuu G. Study of pharmacological activity of traditional mongolian drug Garidi-5. Mongolian Journal of Health Sciences 2013 Vol 10 ( 1), p.111

7. Nantel F, Denis D, Gordon R, Northey A, Cirino M, Metters KM, Chan CC. Distribution and regulation of cyclooxygenase-2 in carrageenan induced inflammation. $\mathrm{Br} \mathrm{J}$ Pharmacol 1999; 128: 853-859.

8. Mohan M, Gulecha VS, Aurangabadkar VM, Balaraman R, Austin A and Thirugnanasampathan S. Analgesic and anti-inflammatory activity of a polyherbal formulation. Oriental Pharmacy and Experimental Medicine 2009; 9(3): 232-237.

9. Silva LMCM, Lima V, Holanda ML, Pinheiro PG,Rodrigues JAG, Lima MEP, Benevides $N M B$ : Antinociceptive and anti-inflammatory activities of lectin from marine red alga Pterocladiella capillacea.Biol Pharm Bull, 2010; 5: 830-835.

10. Santos EN, Lima JCS, Noldin VF, Cechinel-Filho V, Rao VSN, Lima EF, SchmedaHirschmann $G$ et al.:Anti-inflammatory, antinociceptive, and antipyretic effects of methanol extract of Cariniana rubra stem bark in animal models. An Acad Bras Ciênc, 2011; 2: 557-566.

11. Okusada K, Nakamoto K, Nishida M, Fujita-Hamabe W, Kamiya K, Mizushina Y, Satake T, Tokuyama S: The antinociceptive and anti-inflammatory action of the CHCl3soluble phase and its main active component, damnacanthal, isolated from the root of Morindacitrifolia. Biol Pharm Bull, 2011; 1: 103-107.

12. Vinegar R, Schreiber W, Hugo R: Biphasic development of carrageenin oedema in rats. J Pharmacol Exp Ther, 1969; 166:96-103.

13. Matsuda R, Tanihata S: Suppressive effect of sialic acid on the prostaglandin E2mediated oedema in carrageenan induced inflammation of rat hind paws (Japanese). Nippon Yakurigaku Zasshi, 1992; 99: 363-372.

14. Capone ML, Tacconelli S, Rodriguez LG, Patrignani P: NSAIDs and cardiovascular disease: transducing human pharmacology results into clinical read-outs in the general population. Pharmacol Rep, 2010; 62: 518-525. 